\title{
Finer leaf resolution and steeper beam edges using a virtual isocentre in concurrence to PTV-shaped collimators in standard distance - a planning study
}

\author{
Klaus Bratengeier ${ }^{{ }^{*}}$ (D), Barbara Herzog ${ }^{2}$, Sonja Wegener ${ }^{1}$ and Kostyantyn Holubyev ${ }^{3}$
}

\begin{abstract}
Purpose: Investigation of a reduced source to target distance to improve organ at risk sparing during stereotactic irradiation (STX).

Methods: The authors present a planning study with perfectly target-volume adapted collimator compared with multi-leaf collimator (MLC) at reduced source to virtual isocentre distance (SVID) in contrast to normal source to isocentre distance (SID) for stereotactic applications. The role of MLC leaf width and 20-80\% penumbra was examined concerning the healthy tissue sparing. Several prescription schemes and target diameters are considered.

Results: Paddick's gradient index $(\mathrm{Gl})$ as well as comparison of the mean doses to spherical shells at several distances to the target is evaluated. Both emphasize the same results: the healthy tissue sparing in the high dose area around the planning target volume (PTV) is improved at reduced SVID $\leq 70 \mathrm{~cm}$. The effect can be attributed more to steeper penumbra than to finer leaf resolution. Comparing circular collimators at different SVID just as MLC-shaped collimators, always the GI was reduced. Even MLC-shaped collimator at SVID $70 \mathrm{~cm}$ had better healthy tissue sparing than an optimal shaped circular collimator at $S I D 100 \mathrm{~cm}$.

Regarding penumbra changes due to varying SVID, the results of the planning study are underlined by film dosimetry measurements with Agility ${ }^{\mathrm{TM}}$ MLC.
\end{abstract}

Conclusion: Penumbra requires more attention in comparing studies, especially studies using different planning systems. Reduced SVID probably allows usage of conventional MLC for STX-like irradiations.

Keywords: Radiotherapy, Stereotactic Irradiation, Robotic table motion, Multi-leaf collimator, Planning study, Virtual isocentre

\section{Introduction}

\section{General aspects}

Normal linacs are not considered to be suitable for modern stereotactic irradiation (STX). The main reason is the isocentric leaf width of common multi-leaf collimators (MLC). Typical isocentric $0.5 \mathrm{~cm}$ MLCs are assumed too coarse for STX. Leaf widths of the order of $0.3 \mathrm{~cm}$ are recommended by Bortfeld et al. [1]. However, this value is subject to the typical $20-80 \%$ penumbra width of $0.25 \mathrm{~cm}$ to $0.3 \mathrm{~cm}$. Since then, several authors more often consider

\footnotetext{
* Correspondence: Bratengeier_K@ukw.de

'Department of Radiation Oncology, University of Würzburg,

Josef-Schneider-Str. 11, 97080 Würzburg, Germany

Full list of author information is available at the end of the article
}

MLC types and leaf widths [2-7], whereas other parameters which determine the dose gradient, such as the penumbra, are rarely examined or even varied. For example, the authors refer the reader to the method of penumbra control using intermediate energy photons [8] proposed by O'Malley et al. [8] or small-field flattening filters proposed by S.J. Thomas [9] in an older work,.

\section{Virtual isocentre}

The purpose of the present work is to investigate whether a reduced source to target distance combined with MLC (0.5 cm isocentric leaf width) can compete against reference source to isocentre distance $(S I D)$ $100 \mathrm{~cm}$ combined with an ideal round collimator (a limit 
of infinitely narrow leaves). The circular collimator is the ideal shape for spherical targets only. The reduced source to target distance can be realized using, e. g., a virtual isocentre approach (Bratengeier K, Holubyev K, Wegener S: Distance-dependent penumbra: Theoretical considerations and practical implications for stereotactic irradiation using a MLC, submitted): Table positions are a function of the gantry angle and the isocentric table angle in a way that a selected target point is always hit by the beam central axis in a certain fixed distance from the source. This point behaves like an isocentre and is therefore called "virtual isocentre". Its distance from the source is called source to virtual isocentre distance (SVID) which is allowed to differ from $S I D 100 \mathrm{~cm}$. The apparatus as a whole behaves like a linac with changed SID, but however, the radiation head remains unchanged. For the present study we consider the SVID reduced to $70 \mathrm{~cm}$, which is a reasonable distance for head applications, and a SVID of $50 \mathrm{~cm}$ to demonstrate the trends at further reduced SVID. Our special interest is to examine the role of reduced MLC leaf width separately from penumbra effects regarding improved healthy tissue sparing at reduced SVID.

\section{Methods}

\section{Planning system and target definition}

The planning study was performed by means of the therapy planning system (TPS) Philips Pinnacle ${ }^{3_{\mathrm{rax}}}$ version 9.10. A virtual sphere of diameter $20 \mathrm{~cm}$ and physical density $1.0 \mathrm{~g} / \mathrm{cm}^{3}$ was created in TPS on a $0.1 \mathrm{~cm}$ sliced CT. The spherical planning target volume (PTV) was placed in the centre. PTV of diameters $(\varnothing)$ of $1.0 \mathrm{~cm}, 1.3 \mathrm{~cm}$ and $1.7 \mathrm{~cm}$ were created inflating a nearly point size central object using Pinnacle ${ }^{3 \mathrm{rm}}$ expansion functions. Using three different target diameters was intended to investigate the effect of different curvatures. The diameters were chosen small to recognize the effects of MLC induced grating.

\section{Evaluation parameters \\ Mean dose to spherical shells}

To ensure technique-independent characterisation of the dose distribution, concentric spherical shells with borders at $0.3,0.7,1.0,1.3,1.6,1.9,2.2,2.5,2.8,3.0,4.0$, 6.010 .0 and $20.0 \mathrm{~cm}$ were created in the TPS. In the limit of isotropic irradiation, the mean dose at each shell was identical with the dose to an infinitely thin shell of an effective radius $r$ dividing the original shell into two parts of equal volume. The mean dose to the shells was read off in TPS and plotted against the effective radius. In principle, a single beam would be sufficient for the calculation, as mean doses are additive. But a single beam is subject to direction dependent inaccuracies of voxel and slice based planning system and of accidental anisotropies. Therefore, a quasi-isotropic arrangement of sixteen beams was chosen [10] which was equivalent to multi-arc techniques for doses between 100 and $12 \%$ of the maximum dose, as illustrated in Additional file 1.

\section{Conformity index and gradient index}

For further evaluations, Paddick's conformity index PCI [11] and gradient index GI [12] were chosen:

The $P C I$ was defined as

$$
P C I=\left(\frac{V o l(\mathrm{PTV} \cap \mathrm{TV})^{2}}{P T V * T V}\right)_{I C R U}=\left(\frac{T T V^{2}}{P T V * P I V}\right)_{\text {Neurosurg }},
$$

where TV denoted the "treated volume" according to ICRU 83 [13], $T V=\operatorname{Vol}(\mathrm{TV})$ was the volume of this structure. PTV represented the planning target volume to be covered with the desired prescription dose; PTV $\cap$ TV was the intersection of PTV and TV. In the nomenclature of Neurosurgery "TV" is often used as target volume [14]; to avoid confusion with treated volume, here "PTV" was used instead. TTV was the treated target volume and PIV stood for the planning isodose volume ( $=$ treated volume according ICRU $83=$ Referenceisodose enclosed volume).

Paddick's gradient index GI was defined as the ratio between the volume enclosed by the half of the reference dose $P I V_{0.5}$ and $P I V$ :

$$
G I=\frac{P I V_{0.5}}{P I V} .
$$

A GI in stereotactic applications was aimed to be below 3.0 [12].

$V_{66.7 \%}$

Additionally, the volume in which the dose exceeds $66.7 \%$ of the prescription dose, $V_{66.7 \%}$, was evaluated, known as "V12" for 18 Gy prescription in literature. However, within the study the dose to the isocentre was set to the maximum dose of $10 \mathrm{~Gy}$. This choice does not restrict the generality of any results, as the scaling remains free. The prescription was set to the $70 \%-, 80 \%$ and 90\%-isodoses (these situations are called "D70\%", "D80\%", "D90\%"); this means 7 Gy, 8 Gy or 9 Gy, respectively, were aimed to surround the PTV exactly in a way described below.

\section{Planning details}

The MLC leaves were positioned using the "ExposePTV" block margin function of the Pinnacle ${ }^{3}$ planning system in such a way that the MLC leaves touched the margin around the PTV in beam eye view projection. Also beams with PTV conformal, nearly circular blocks were created using the margin. The same margin was 
used for all beams of a given technique. To study the influence of prescription, the $70 \%$-, $80 \%$ - and $90 \%$-doses of the reference isocentre dose $(=100 \%)$ were chosen as "PTV-surrounding", respectively. They cover at least 99\% of the PTV. A block thickness was chosen to produce the same transmission as the standard Elekta Agility ${ }^{\mathrm{TM}}$ MLC with $0.5 \mathrm{~cm}$ leaf width at $S I D 100 \mathrm{~cm}$. To carve out the impact of SVID and consequential effects of the penumbra changes, same conditions for block and MLC were simulated; the virtual block was placed in the MLC distance. Thus, the block behaved like a MLC with infinitely narrow leaves. To compare the techniques with each other, the same dose was prescribed to the isocentre; the PTV margin was iteratively adapted to achieve the same mean dose inside the PTV. Details of this method are described in Additional file 2. Changes of the penumbra were simulated exemplarily by a superposition of beams with different diameters. Beam charact eristics (profiles) were determined according to Additional file 3.

\section{Results}

The authors considered deviances of $\pm 2 \%$ of $P C I$ as "nearly equal"; effects of similar order could occur due to dose grid effects and effects of digitization (voxel generation) of a spherical object (see [15] for volume effects of structure definition). Thus, only for a PTV diameter of $1.0 \mathrm{~cm}$ and a $S I D$ of $100 \mathrm{~cm}$, the $P C I$ differed markedly for MLC compared with circular collimator (Table 1).

Reduced SVID influenced both, penumbra and effective leaf width. The following sections were conceived to separate penumbra and lead width effects.

\section{SID 100 vs. SVID 70/50}

The GI (Table 2) always decreased for reduced SVID, for all target diameters and prescriptions. It fell below the critical value of 3.0 for $\mathrm{D} 70 \%, \varnothing 1.3 \mathrm{~cm}$ and $\mathrm{D} 80 \%, \varnothing 1.7 \mathrm{~cm}$.

In more detail, the effect of reduced SVID on a radial dose distribution is shown Figs. 1 and 2 for circular collimator and MLC, respectively. The distributions were normalized to that one at $S I D 100 \mathrm{~cm}$. For both, circular collimator and MLC, the decrease of the dose to the surrounding healthy tissue at the reduced SVID was almost independent from the prescription. However, the effect of healthy tissue sparing utilizing reduced SVID was more pronounced for the "MLC" situation (Fig. 2); most probably due to the additional effect of finer approximations of a circular beam formed by thinner MLC leaves. The next section is intended to quantify the MLC and penumbra contributions.

\section{Circular collimator vs. MLC}

The gradient index in Table 2 clearly showed lower GI values for circular collimators with respect to MLC. Accordingly, Fig. 3 compares the dose distributions for a MLC and a circular collimator at the same SVID. Clearly, the MLC always led to an additional dose in the surrounding healthy tissue. This additional contribution decreased at reduced SVID. This decrease was not always most effective for SVID 50, assumedly due to"digitization" effects of the circular shape by slots of finite width. Obviously, the influence of MLC-beam-shaping was less than the distance effects shown in Fig. 1.

\section{MLC at SVID 70/50 or optimal shape at SID 100?}

Can a reduced SVID compensate for the "roughness" of MLC beam shaping? In four of the five cases listed in Table 2, the GI for the MLC at SVID $70 \mathrm{~cm}$ was lower than ideal beam shaping by a circular collimator at SID $100 \mathrm{~cm}$ (and in the one remaining case, the values differed only marginally). Clearly, the steeper penumbra at reduced SVID overcompensated the deteriorating effect of MLC beam shaping even for the $0.5 \mathrm{~cm}$ MLC. In more detail, the cumulative effect of combining reduced SVID with MLC can be deduced from Fig. 4, which shows the MLC dose distributions normalized to that of circular collimator at standard $S I D 100 \mathrm{~cm}$. In any case for the high dose region (hence, up to double PTV radius), using a MLC at $S V I D 70 \mathrm{~cm}$ was preferable to a perfect beam shaper (using appropriate circular collimators of optimized diameter) at $S I D 100 \mathrm{~cm}$.

Table 1 Paddick's conformity index PCI for investigated constellations

\begin{tabular}{|c|c|c|c|c|c|c|c|c|}
\hline \multirow[t]{2}{*}{$P C l$} & & \multirow[b]{2}{*}{ SID or SVID $[\mathrm{cm}]$} & \multicolumn{3}{|c|}{ Circular Collimator } & \multicolumn{3}{|c|}{ MLC $0.5 \mathrm{~cm}$ (isocentric) } \\
\hline & & & 100 & 70 & 50 & 100 & 70 & 50 \\
\hline$\varnothing[\mathrm{cm}]$ & Prescription & & & & & & & \\
\hline 1.0 & D80\% & & 0.87 & 0.92 & 0.92 & 0.79 & 0.88 & 0.94 \\
\hline 1.3 & D70\% & & 0.88 & 0.92 & 0.93 & 0.85 & 0.89 & 0.93 \\
\hline 1.3 & D80\% & & 0.87 & 0.89 & 0.90 & 0.82 & 0.88 & 0.90 \\
\hline 1.3 & D90\% & & 0.85 & 0.83 & 0.84 & 0.85 & 0.86 & 0.86 \\
\hline 1.7 & D80\% & & 0.91 & 0.92 & 0.93 & 0.85 & 0.92 & 0.92 \\
\hline
\end{tabular}


Table 2 Gradient index Gl for investigated constellations

\begin{tabular}{|c|c|c|c|c|c|c|c|c|}
\hline \multirow[t]{2}{*}{ Gl } & & \multirow[b]{2}{*}{ SID or SVID [cm] } & \multicolumn{3}{|c|}{ Circular Collimator } & \multicolumn{3}{|c|}{ MLC $0.5 \mathrm{~cm}$ (isocentric) } \\
\hline & & & 100 & 70 & 50 & 100 & 70 & 50 \\
\hline$\varnothing[\mathrm{cm}]$ & Prescription & & & & & & & \\
\hline 1.0 & D80\% & & 3.90 & 3.45 & 3.20 & 4.21 & 3.74 & 3.63 \\
\hline 1.3 & D70\% & & 2.91 & 2.70 & 2.61 & 3.15 & 2.92 & 2.77 \\
\hline 1.3 & D80\% & & 3.26 & 2.83 & 2.78 & 3.57 & 3.22 & 3.01 \\
\hline 1.3 & D90\% & & 4.19 & 3.69 & 2.44 & 4.65 & 4.05 & 3.73 \\
\hline 1.7 & D80\% & & 2.86 & 2.63 & 2.52 & 3.15 & 2.85 & 2.69 \\
\hline
\end{tabular}

\section{Clinical relevance?}

All results from sections $\mathrm{A}, \mathrm{B}, \mathrm{C}$ were also reflected in Table 3. For a MLC, $\mathrm{V}_{66.7 \%}$ was reduced from $13 \%$ up to $17 \%$, if $S I D 100 \mathrm{~cm}$ was reduced to SVID $70 \mathrm{~cm}$. For $\varnothing$ $1.0 \mathrm{~cm}$ this means a reduction of more than half of the target volume; even for $\varnothing 1.7 \mathrm{~cm}$ it resulted in a reduction of one third. If a circular collimator at SID $100 \mathrm{~cm}$ was replaced by a MLC at $S$ ID $70 \mathrm{~cm}, \mathrm{~V}_{66.7 \%}$ was still reduced by $11 \pm 4 \%$. Aspects of the clinical relevance of these results were also shown by an example in Additional file 4.

\section{Effects of superposition and penumbra increase}

To evaluate the increase in the healthy tissue dose resulting from an enlarged penumbra for combined beam arrangements, we artificially raised the penumbra at constant SID using a superposition of beams with differing diameters.

As a reference, we considered a PTV $\varnothing$ of $1.0 \mathrm{~cm}$ and used circular collimator with a margin around PTV to meet the demands of a D80\% prescription (Fig. 5). We considered superposition of pairwise wider and narrower beams, identical for the whole arrangement. Two cases were generated with PTV margins of additional $+0.10 \mathrm{~cm}$ and $-0.10 \mathrm{~cm}($ case $\alpha$ ), and $+0.15 \mathrm{~cm}$ and $-0.05 \mathrm{~cm}$ (case $\beta)$. The same prescription was used for both cases. The relative beam weights were adjusted to achieve the same mean dose to the PTV (71.5 and 28.5\%, respectively, for $\alpha$, 35.4 and $64.6 \%$ for $\beta$ ). We found that for $0.20 \mathrm{~cm}$ difference between PTV margins the penumbra increased from $0.35 \mathrm{~cm}$ (reference) to $0.37 \mathrm{~cm}(\alpha)$ or $0.38 \mathrm{~cm}(\beta)$. The associated increase of the healthy tissue dose reached its maximum of about $7 \%$ at a distance of $0.5 \mathrm{~cm}$ from the PTV, for both $\alpha$ and $\beta$, see Fig. 5. The penumbra increase depended on the difference between margins only. Assuming this dependence to be linear, we estimated the systematic increase of the healthy tissue dose at SID $100 \mathrm{~cm}$ for a margin step $0.02 \mathrm{~cm}$ not to exceed $0.7 \%$. At SVID $70 \mathrm{~cm}$ for margin step $0.04 \mathrm{~cm}$ it did not exceed $1.4 \%$, and at SVID $50 \mathrm{~cm}$ for margin step $0.10 \mathrm{~cm} 3.5 \%$. Thus, combining beams to achieve the prescribed radial mean PTV dose (see Additional file 1 and methods

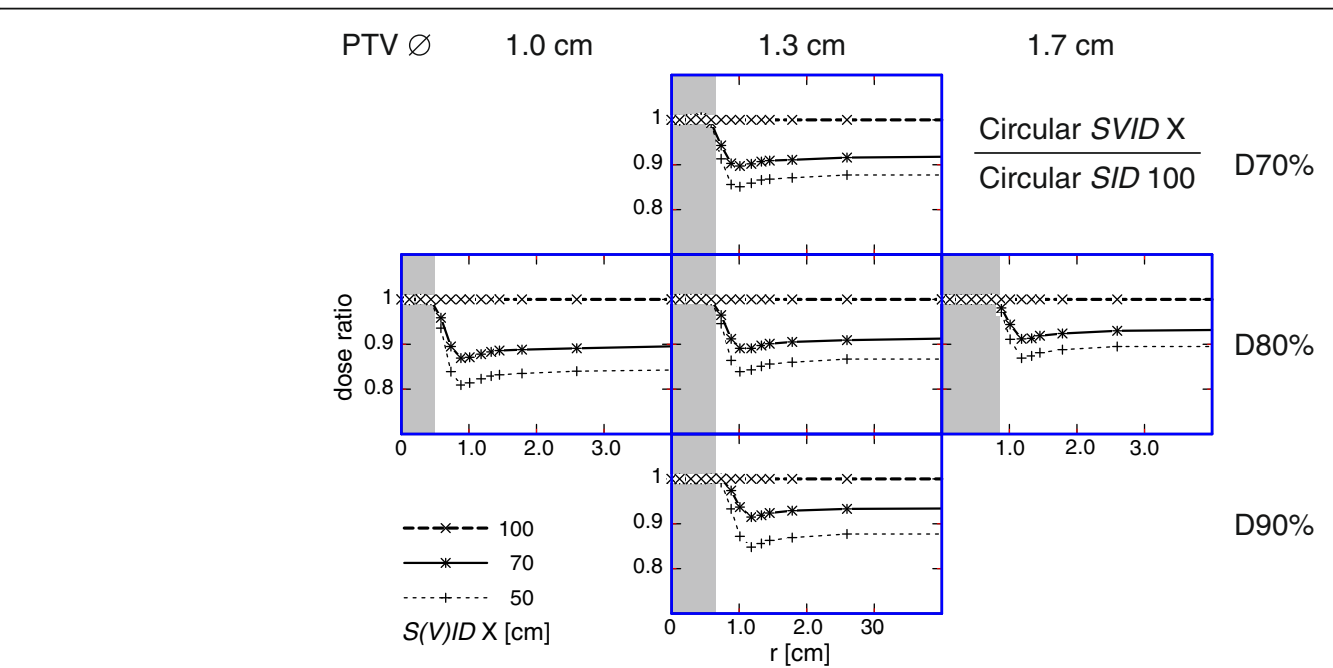

Fig. 1 Circular collimator: SVID dependence of radial dose. Radial dose distribution for circular collimator at SVID $70 \mathrm{~cm}$ (continuous) and at $50 \mathrm{~cm}$ (thin dashed), normalized to the distribution for circular collimator at SID $100 \mathrm{~cm}$. Shaded area: PTV. Left: PTV $\varnothing 1.0 \mathrm{~cm} ;$ middle: PTV $\varnothing$ $1.3 \mathrm{~cm}$; right: PTV $\varnothing 1.7 \mathrm{~cm}$. Prescriptions: top: D70\%; middle: D80\%; bottom: D90\%. S(V)ID: SID or SVID 


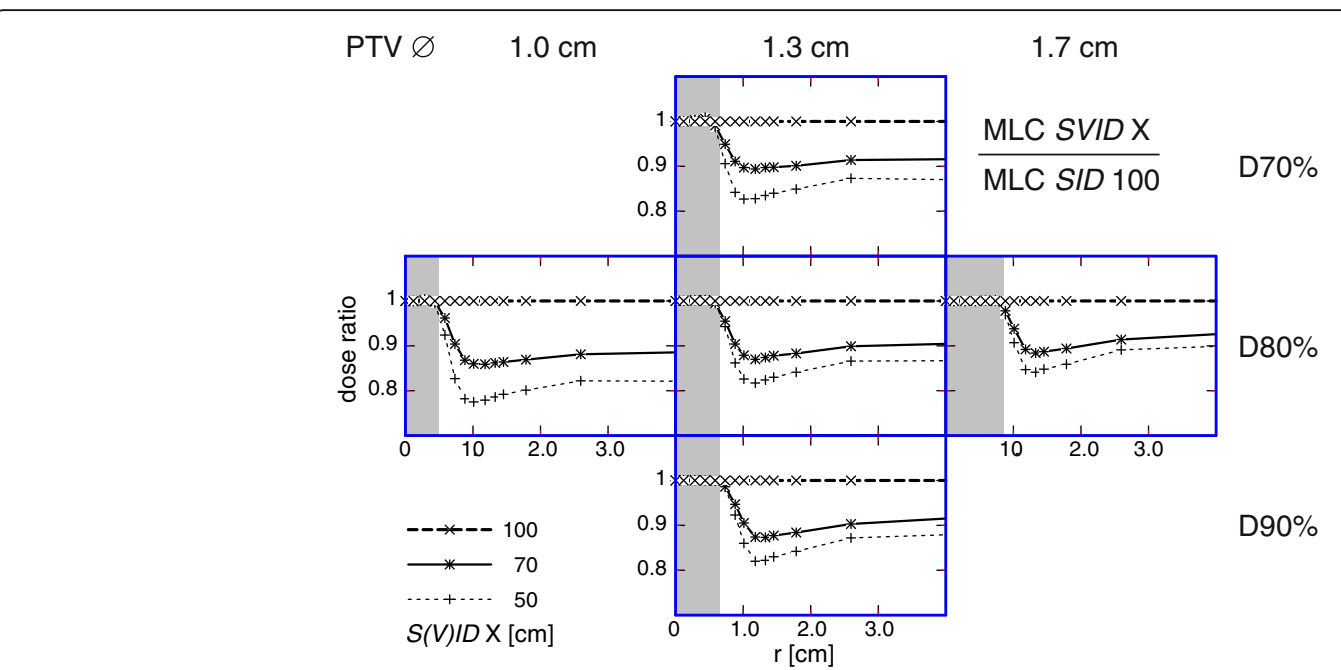

Fig. 2 MLC: SVID dependence of radial dose. Radial dose distribution for MLC at SVID $70 \mathrm{~cm}$ (continuous) and $50 \mathrm{~cm}$ (thin dashed), normalized to the distribution for MLC at SID $100 \mathrm{~cm}$. Shaded area: PTV. Left: PTV $\varnothing 1.0 \mathrm{~cm}$; middle: PTV $\varnothing 1.3 \mathrm{~cm}$; right: PTV $\varnothing 1.7 \mathrm{~cm}$. Prescriptions: top: D70\%; middle: D80\%; bottom: D90\%. S(V)ID: SID or SVID

section) did not influence the results for the healthy tissue at $S I D 100 \mathrm{~cm}$ and SVID $70 \mathrm{~cm}$. For SVID $50 \mathrm{~cm}$, used for demonstration purposes, the systematic dose increase from beam superposition did not exceed $3.5 \%$.

\section{Discussion}

\section{MLC beam shaping}

Not surprisingly, the use of a MLC instead of optimal circular collimators leads to more dose to the healthy tissue around the PTV, see Fig. 3. However, the effect of MLC is less pronounced than intuitively expected. At SID $100 \mathrm{~cm}$ the MLC provides coarser approximation of the circular collimator as at SVID $70 \mathrm{~cm}$ or even $50 \mathrm{~cm}$. In the worst case of $1.0 \mathrm{~cm}$ diameter spherical PTV, a
$1.0 \mathrm{~cm} \times 1.0 \mathrm{~cm}$ square MLC approximation leaves 27\% of the area around the PTV unblocked, if the two MLC leaves, at both sides each, touch the PTV in the beam projection. For SVID $50 \mathrm{~cm}$ the unblocked area would numerically be $19 \%$ for four leaves at both sided of a circle. Interestingly, the planning study shows that the effect of MLC vs circular collimator (Fig. 3) increases the dose to the healthy tissue only by half of that, $10-13 \%$. This is due to the fact that, depending on prescription, the MLC apertures have to be generated from negative PTV margins [16] and thus not always fully contribute to the primary dose in healthy tissue. In contrast, the penumbra in any case contributes to the primary dose in the healthy tissue, so its effect seems to be always larger.

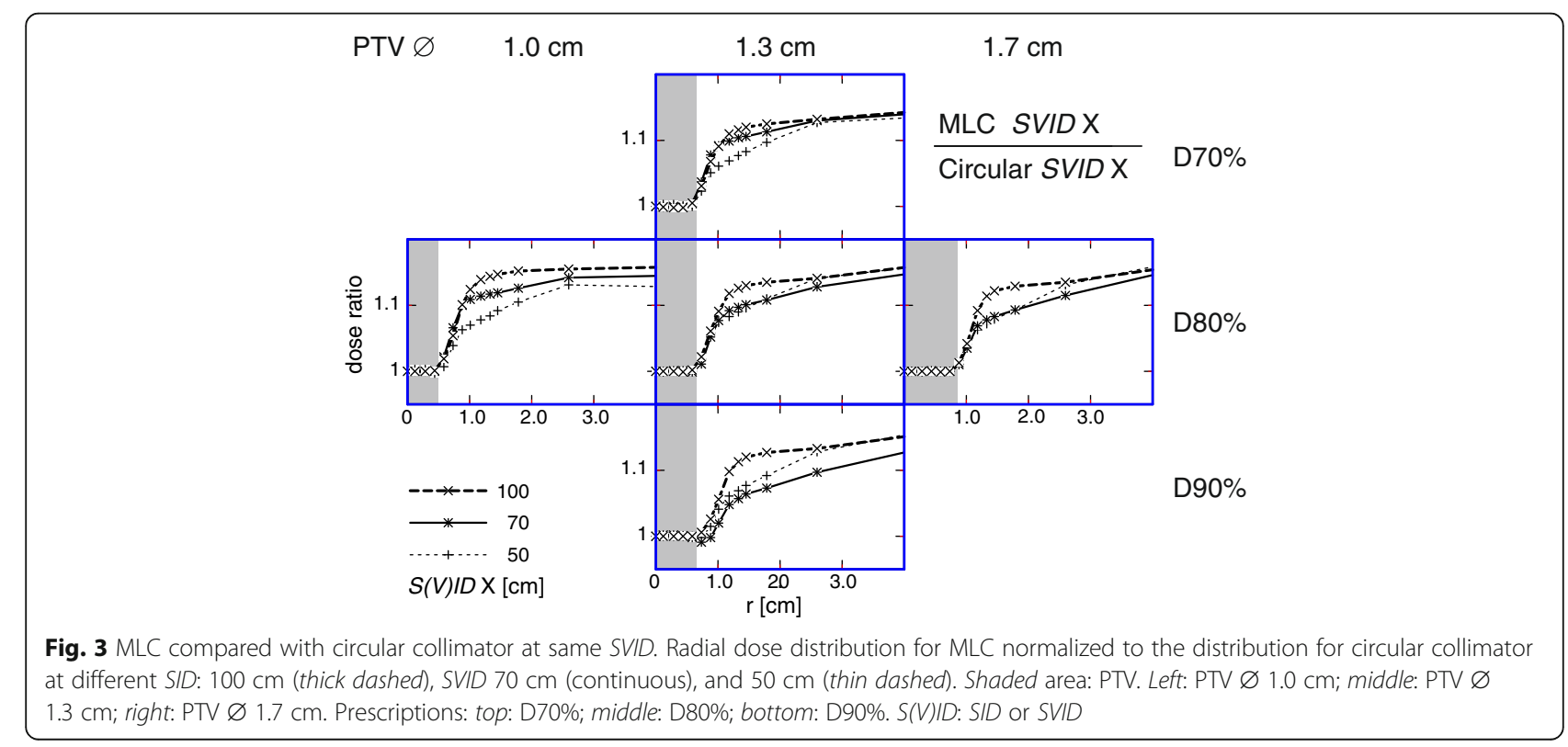




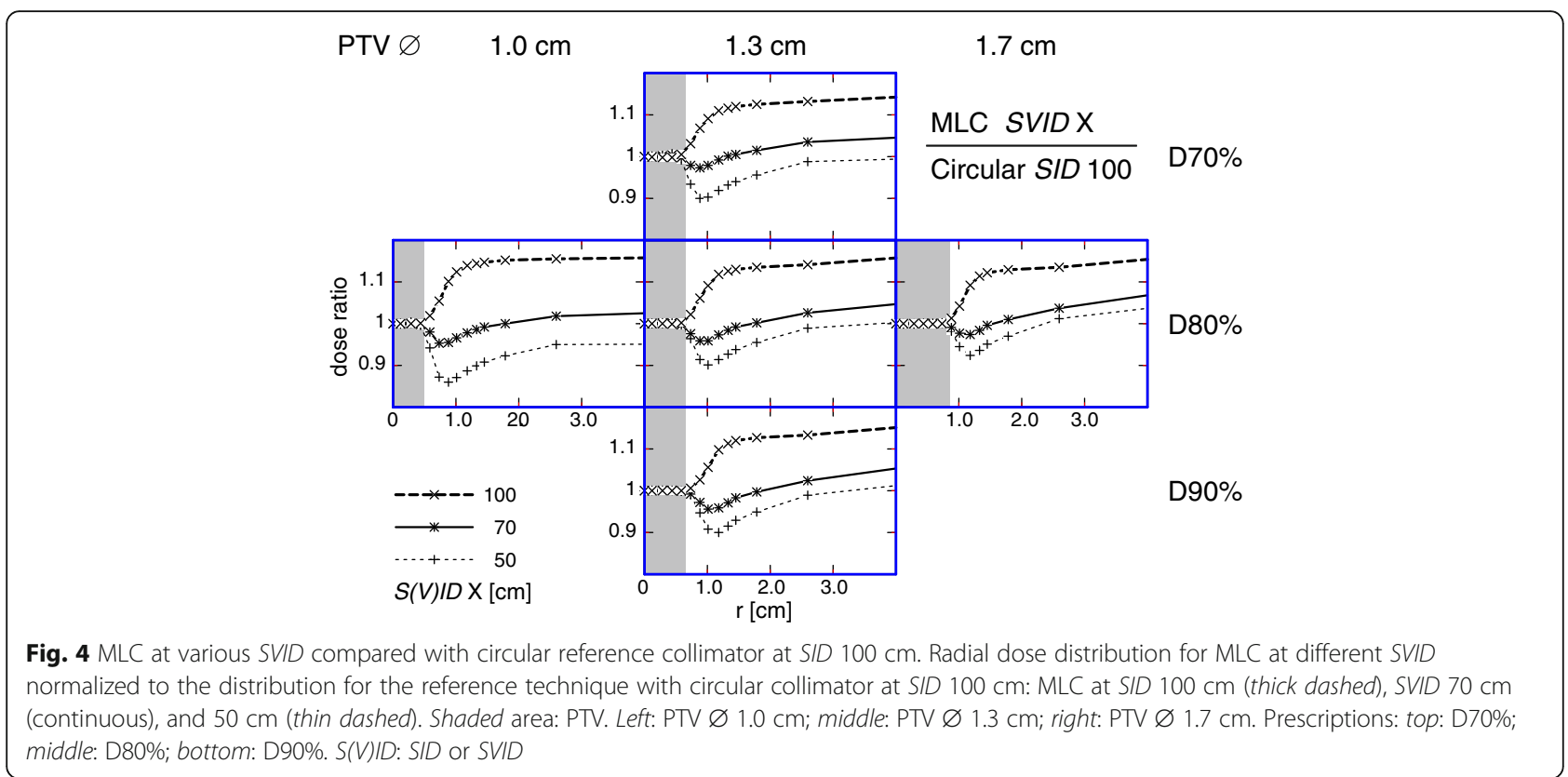

The deterioration of the dose falloff from MLC apertures can be overcompensated by a reduced SVID, as demonstrated in Fig. 4. In the high dose area adjacent to the PTV, the $S V I D \leq 70 \mathrm{~cm}$ combined with MLC performs better than the perfectly adapted circular collimator at standard SID $100 \mathrm{~cm}$. The effect must be traced back to the steeper penumbra as depicted in Fig. 6 and quantified in Fig. 5. An increase of $20-80 \%$ penumbra width by 0.02 or $0.03 \mathrm{~cm}$ leads to $7 \%$ larger healthy tissue dose. For the MLC as modelled in the planning system SID $100 \mathrm{~cm}$ compared to SVID $70 \mathrm{~cm}$ leads to penumbra increase of $0.03 \mathrm{~cm}$ in J direction, $0.08 \mathrm{~cm}$ in L direction. This is semiquantitatively in good agreement with the increase of the healthy tissue dose of about $12-14 \%$ seen in Fig. 2 (D80\%, Ø $1.3 \mathrm{~cm})$.

\section{Validity of the planning study results}

Note that the conclusions above probably are quite general and independent from the beam shaping device, although a special (spherical) PTV shape was chosen. The present study was performed for small spherical targets of different diameters. These targets stand for objects with various curvatures. The simple circular shape allows the definition of an optimal circular aperture, which is regarded as the limit of an infinitely small leaf width. All results were qualitatively independent from the PTV diameter. They were also independent from different prescription schemes, be that D70\%, D80\% or D90\%.

\section{The role of penumbra steepness}

For circular collimator (Fig. 1) combined with reduced $S V I D$, the improved healthy tissue sparing comes from the penumbra decrease. For MLC, the improvement in healthy tissue sparing at reduced SVID (Fig. 2) is dominated by the penumbra decrease, which can be inferred by comparing Fig. 2 vs. Fig. 1 and can be concluded from Fig. 4. As long as penumbra is decreased by $0.02 \mathrm{~cm}$ or more at reduced SVID, the MLC performs better than an ideal collimator at standard SID $100 \mathrm{~cm}$. Although this was shown for the Agility ${ }^{\mathrm{TM}}$ head, this

Table 3 Volume of $66.7 \%\left(V_{66.7 \%}\right)$ of prescription dose for investigated constellations

\begin{tabular}{|c|c|c|c|c|c|c|c|c|}
\hline \multirow[t]{2}{*}{$V_{66.7 \%}$} & & \multirow[b]{2}{*}{ SID or SVID $[\mathrm{cm}]$} & \multicolumn{3}{|c|}{ Circular Collimator } & \multicolumn{3}{|c|}{ MLC $0.5 \mathrm{~cm}$ (isocentric) } \\
\hline & & & 100 & 70 & 50 & 100 & 70 & 50 \\
\hline$\varnothing[\mathrm{cm}]$ & Prescription & & & & & & & \\
\hline 1.0 & D80\% & & 1.87 & 1.58 & 1.44 & 2.09 & 1.73 & 1.50 \\
\hline 1.3 & D70\% & & 3.01 & 2.62 & 2.44 & 3.30 & 2.87 & 2.57 \\
\hline 1.3 & D80\% & & 3.58 & 3.12 & 2.89 & 3.98 & 3.34 & 3.11 \\
\hline 1.3 & D90\% & & 4.62 & 4.22 & 3.88 & 5.06 & 4.28 & 4.00 \\
\hline 1.7 & D80\% & & 6.34 & 5.72 & 5.38 & 7.05 & 6.08 & 5.67 \\
\hline
\end{tabular}




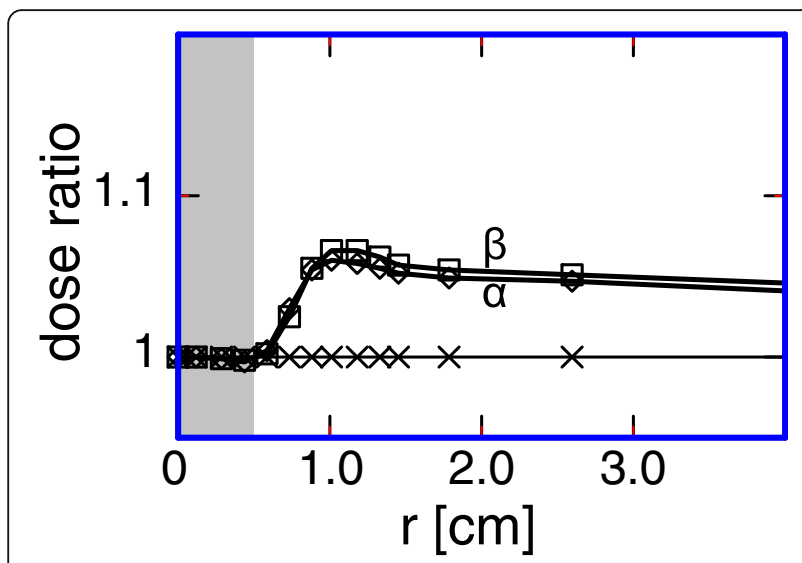

Fig. 5 Simulation of enlarged penumbra. Radial dose distribution for superimposed beam arrangements normalized to reference beam arrangement generated using PTV margin. Shaded area: PTV. Crosses: Reference (PTV $\varnothing=1.0 \mathrm{~cm}, \mathrm{D} 80 \%$, circular collimator, PTV margin $0.00 \mathrm{~cm}$ ). Diamonds: superposition a (generated from PTV margins additionally $-0.10 \mathrm{~cm}$ and $+0.10 \mathrm{~cm}$, respectively). Squares: superposition $\beta$ (generated from PTV margins additionally $+0.15 \mathrm{~cm}$ and $-0.05 \mathrm{~cm}$, respectively). See text for explanation

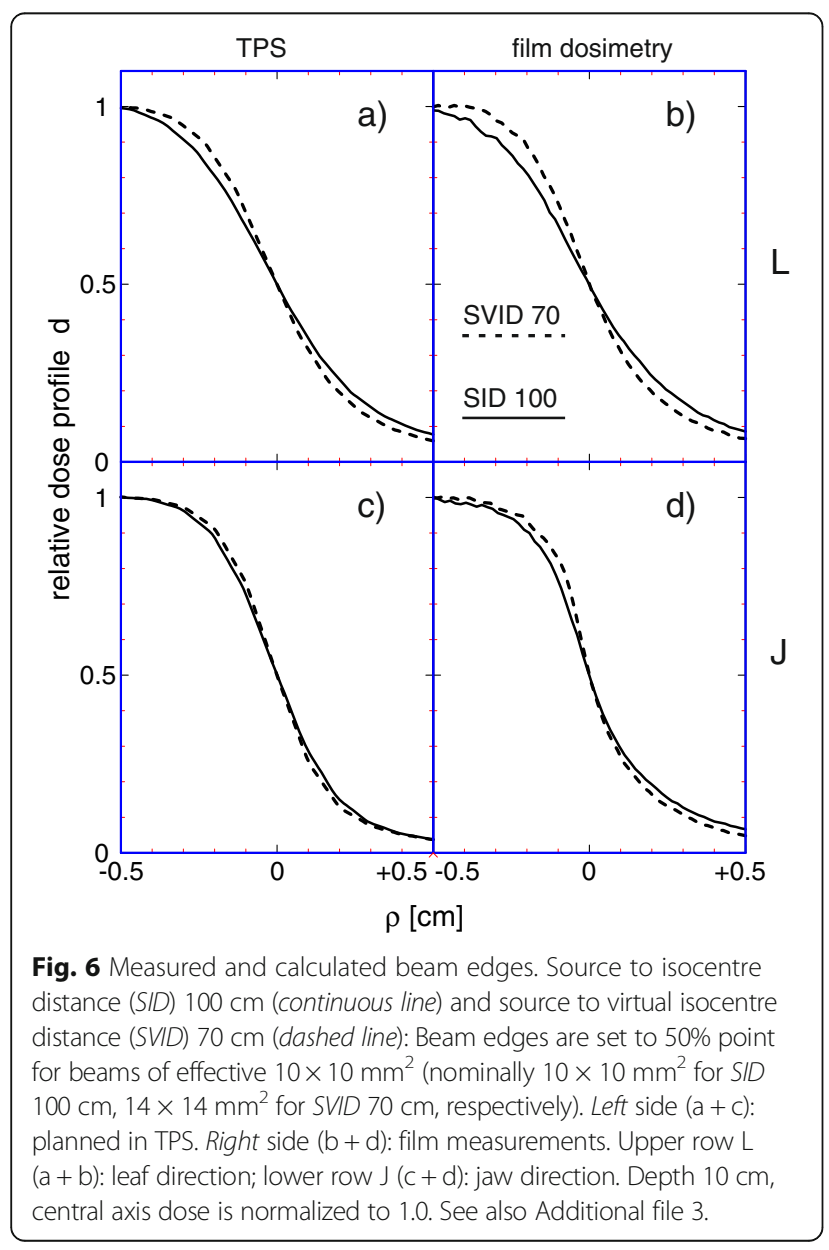

result can be assumed relevant also for other MLCs, as the effect can be traced back to the geometrical penumbra. Independent from the present planning study, the importance of the penumbra can be deduced from more theoretical considerations (Bratengeier K, Holubyev K, Wegener S: Distance-dependent penumbra: Theoretical considerations and practical implications for stereotactic irradiation using a MLC, submitted).

In this study, the healthy tissue sparing effect of reduced SVID decreases for a larger PTV radius independently of beam shaping device, see Figs. 1 and 2. The sparing effect is dominated by penumbra decrease, which becomes less important for larger PTV: the volume of a thin layer around PTV, where penumbra dose dominates, becomes smaller relative to the PTV volume itself.

\section{Advantages of reduced SVID}

The effect of using MLC instead of ideal circular collimator (Fig. 3) is found (almost independent of SVID and prescription) at the level of $10 \%$ dose increase to the healthy tissue. The effect of reduced SVID is proven for SVID $70 \mathrm{~cm}$, practical for head irradiations, at the level of $10 \%$ dose decrease due to decreased penumbra. Thus, at SVID $70 \mathrm{~cm}$ combined with MLC the two effects compensate and the plan quality is at least as good as at SID $100 \mathrm{~cm}$ combined with circular collimator (Fig. 4). In fact, the additional improved beam shaping due to narrower effective leaf widths ensures even further healthy tissue sparing.

\section{Relation to neurosurgical literature data}

The increase of quality by using SVID $70 \mathrm{~cm}$ or less is clearly significant and relevant: Dose to the surrounding decreases, as can be seen from GI and $V_{66.7 \%}$.

The GI for higher dose maxima and for more extended targets decreases below the intended GI $<3.0$ as demanded by the radiosurgery consortium [14], even for the $0.5 \mathrm{~cm}$ MLC at SVID 70 and below. For targets of $1.0 \mathrm{~cm}$ diameter, D50\% should be used instead of D70\%, because Paddick and Lippitz demonstrated a strong decrease of GI with increasing maximum dose [12] for typical beam profiles.

The presented results even assume infinitely variable collimator diameters, which will not be available for fixed sets of applicators of different diameters. In contrast, MLC and jaw can be steered in sub-millimeter range, as in the presented study. This fact additionally favours the MLC-SVID-approach. This is all the more the case for the shaping of non-spherical targets. Certainly, shaping by a MLC will be even more advantageous compared to circular applicators, if non-spherical targets are to be treated: the application time may be 
reduced. The treatment time is further decreased as a result of the inverse-quadratic law.

Recently, several authors pushed dynamic techniques using table movement [17] or complex leaf steering (see i.e., [18]) that require fail-safe and precise table and leaf movements, much more than needed for the present study. Therefore we excluded safety and precision aspects for the present study.

\section{Conclusion}

Using reduced SVID (e.g., in form of virtual isocentre) in combination with beam shaping device of any kind reduces the penumbra. Decreased penumbra provides an important contribution to the sparing of surrounding healthy tissue. The penumbra decrease obviously accounts for better healthy tissue sparing at reduced SVID. The present work proves that this effect dominates the decrease of healthy tissue dose at reduced SVID combined with MLC. In summary, for an Elekta Agility head, even a $0.5 \mathrm{~cm}$ leaf MLC at SVID $70 \mathrm{~cm}$ distance allows at least as good or even better healthy tissue sparing as an optimally shaped collimator at $S I D 100 \mathrm{~cm}$.

In summary, using the SVID in reduced distance mode, the MLC-techniques could deliver approximately equal or better plan quality due to overcompensation of MLC "roughness" by the reduced penumbra.

The authors are convinced that the penumbra requires more attention in comparing studies, especially studies using different planning systems. This view is supported by theoretical work presently considered for publishing (Bratengeier K, Holubyev K, Wegener S: Distancedependent penumbra: Theoretical considerations and practical implications for stereotactic irradiation using a MLC, submitted). Guidelines for stereotactic irradiation should not only define the leaf width but should also contain requirements for the penumbra [19].

Further studies should address more complex patient related targets and other types of linacs or sources combined with a virtual isocentre and its variable source-to-patient distances.

\section{Additional files}

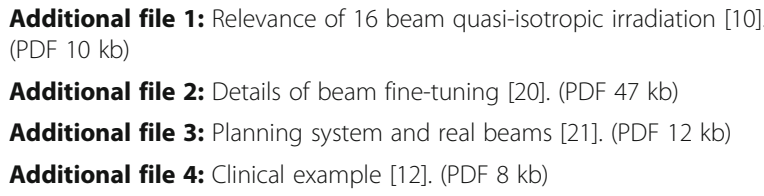

\section{Acknowledgements}

Many thanks to Johannes Greber for proofreading and to Otto Sauer for helpful discussions.

\section{Funding}

This publication was supported by the Open Access Publication Fund of the University of Wuerzburg.

\section{Availability of data and materials}

Not applicable.

\section{Authors' contributions}

$\mathrm{KB}$ designed the study and performed most of the calculations and was responsible for the theoretical considerations. SW contributed the film dosimetry of real beams. $\mathrm{BH}$ performed parts of the planning study. $\mathrm{KH}$ discussed the study. All authors read and approved the final manuscript.

\section{Competing interests}

The authors declare that they have no competing interests.

\section{Consent for publication}

Not applicable.

\section{Ethics approval and consent to participate}

Not applicable.

\section{Publisher's Note}

Springer Nature remains neutral with regard to jurisdictional claims in published maps and institutional affiliations.

\section{Author details}

${ }^{1}$ Department of Radiation Oncology, University of Würzburg, Josef-Schneider-Str. 11, 97080 Würzburg, Germany. ${ }^{2}$ Martin-Luther-Universität Halle-Wittenberg, Institute of Physics, Von-Danckelmann-Platz 3, 06120 Halle (Saale), Germany. ${ }^{3}$ University of Freiburg, Klinik für Strahlenheilkunde, Abt. Medizinische Physik, Robert-Koch-Str. 3, 79106 Freiburg, Germany.

Received: 13 March 2017 Accepted: 16 May 2017

Published online: 25 May 2017

\section{References}

1. Bortfeld T, Oelfke U, Nill S. What is the optimum leaf width of a multileaf collimator? Med Phys. 2000;27:2494-502.

2. Ohtakara K, Hayashi S, Tanaka H, Hoshi H. Dosimetric comparison of $2.5 \mathrm{~mm}$ vs. $3.0 \mathrm{~mm}$ leaf width micro-multileaf collimator-based treatment systems for intracranial stereotactic radiosurgery using dynamic conformal arcs: implications for treatment planning. Jpn J Radiol. 2011;29:630-8.

3. Monk JE, Perks JR, Doughty D, Plowman PN. Comparison of a micromultileaf collimator with a 5-mm-leaf-width collimator for intracranial stereotactic radiotherapy. Int J Radiat Oncol Biol Phys. 2003:57:1443-9.

4. Tanyi JA, Kato CM, Chen Y, Chen Z, Fuss M. Impact of the high-definition multileaf collimator on linear accelerator-based intracranial stereotactic radiosurgery. Br J Radiol. 2011;84:629-38.

5. Dvorak P, Georg D, Bogner J, Kroupa B, Dieckmann K, Potter R. Impact of IMRT and leaf width on stereotactic body radiotherapy of liver and lung lesions. Int J Radiat Oncol Biol Phys. 2005;61:1572-81.

6. Jin JY, Yin FF, Ryu S, Ajlouni M, Kim JH. Dosimetric study using different leaf-width MLCs for treatment planning of dynamic conformal arcs and intensity-modulated radiosurgery. Med Phys. 2005;32:405-11.

7. Dhabaan A, Elder E, Schreibmann E, Crocker I, Curran WJ, Oyesiku NM, Shu HK, Fox T. Dosimetric performance of the new high-definition multileaf collimator for intracranial stereotactic radiosurgery. J Appl Clin Med Phys. 2010;11:3040.

8. O'Malley L, Pignol JP, Beachey DJ, Keller BM, Presutti J, Sharpe M. Improvement of radiological penumbra using intermediate energy photons (IEP) for stereotactic radiosurgery. Phys Med Biol. 2006;51:2537-48.

9. Thomas SJ. Factors affecting penumbral shape and 3D dose distributions in stereotactic radiotherapy. Phys Med Biol. 1994;39:761-71.

10. Bratengeier K, Seubert B, Holubyev K, Schachner H. Considerations on IMRT for quasi-isotropic non-coplanar irradiation. Phys Med Biol. 2012;57:7303-15.

11. Paddick I. A simple scoring ratio to index the conformity of radiosurgical treatment plans. Technical note. J Neurosurg. 2000;93 Suppl 3:219-22.

12. Paddick I, Lippitz B. A simple dose gradient measurement tool to complement the conformity index. J Neurosurg. 2006;105 Suppl:194-201.

13. ICRU, "Prescribing, Recording, and Reporting Photon-Beam IntensityModulated Radiation Therapy (IMRT): Contents," J ICRU 10, NP (2010). 
14. Torrens M, Chung C, Chung HT, Hanssens P, Jaffray D, Kemeny A, Larson D, Levivier M, Lindquist C, Lippitz B, Novotny Jr J, Paddick I, Prasad D, Yu CP. Standardization of terminology in stereotactic radiosurgery: Report from the Standardization Committee of the International Leksell Gamma Knife Society: special topic. J Neurosurg. 2014;121 Suppl:2-15.

15. Prabhakar R, Rath GK, Haresh KP, Manoharan N, Laviraj MA, Rajendran M, Julka PK. A study on the tumor volume computation between different 3D treatment planning systems in radiotherapy. J Cancer Res Ther. 2011;7:168-73.

16. Adams EJ, Cosgrove VP, Shepherd SF, Warrington AP, Bedford JL, Mubata CD, Bidmead AM, Brada M. Comparison of a multi-leaf collimator with conformal blocks for the delivery of stereotactically guided conformal radiotherapy. Radiother Oncol. 1999;51:205-9.

17. Wilson B, Otto K, Gete E. A simple and robust trajectory-based stereotactic radiosurgery treatment. Med Phys. 2017;44:240-8.

18. Thomas EM, Popple RA, Markert JM, Fiveash JB. In reply: volumetric arc therapy (RapidArc) vs Gamma Knife radiosurgery for multiple brain metastases. Neurosurgery. 2015;76:E353-4.

19. Kocher M, Wittig A, Piroth MD, Treuer H, Seegenschmiedt H, Ruge M, Grosu AL, Guckenberger M. Stereotactic radiosurgery for treatment of brain metastases. A report of the DEGRO Working Group on Stereotactic Radiotherapy. Strahlenther Onkol. 2014;190:521-32.

20. Fenner J, Gwilliam M, Mehrem R, Bird A, Walton L. Analytical description of dose profile behaviour in Gamma Knife radiosurgery. Phys Med Biol. 2008;53:2035-49.

21. Micke A, Lewis DF, Yu X. Multichannel film dosimetry with nonuniformity correction. Med Phys. 2011;38:2523-34.

\section{Submit your next manuscript to BioMed Central and we will help you at every step:}

- We accept pre-submission inquiries

- Our selector tool helps you to find the most relevant journal

- We provide round the clock customer support

- Convenient online submission

- Thorough peer review

- Inclusion in PubMed and all major indexing services

- Maximum visibility for your research

Submit your manuscript at www.biomedcentral.com/submit 